\title{
Systemtechnische Ansätze zur Erfüllung neuer Anforderungen an Lagersysteme
}

\author{
DR.-ING. THORSTEN SCHMIDT \\ FRAUNHOFER-INSTITUT FÜR MATERIALFLUSS UND LOGISTIK IML, DORTMUND
}

Zukünftigen Lagersystemen werden aufgrund gewandelter Anforderungen Eigenschaften abverlangt, die mitunter in einem Widerspruch zueinander stehen. Im Folgenden sollen dazu betrieblichtechnische Anforderungen und systemtechnische Lösungsansätze analysiert werden.

Due to changing requirements for storage systems, future storage solutions have to meet partially contradicting characteristics. This article analyses such requirements from a organisational-technical viewpoint and looks into approaches to meet those challenges.

\section{Zukünftige Anforderungen an den effizienten Lagerbetrieb}

Die Systemauswahl eines Lagersystems wird grundsätzlich vom speziellen Anwendungsfall bestimmt. Neben den klassischen, für den jeweiligen Spezialfall dominierenden Einflussparametern, wie z.B. Artikel- und Auftragsspektrum, prägen ebenso externe Einflüsse und Vorgaben den Auswahl- und Gestaltungsprozess der Lagerplanung. So stellen ggf. verschärfte Anforderungen an die Chargenverwaltung (Stichwort EU-Richtlinie 178/02-Rückverfolgbarkeit) auch zusätzliche Anforderungen an die eingesetzte Lagertechnik. Die Forderungen hinsichtlich Transparenz und Flexibilität stellen deshalb immer wichtigere Faktoren dar. Unter Flexibilität wird sowohl die Einsatzflexibilität für unterschiedliche, ggf. sich im Laufe der Nutzung ändernde Einsatzspektren als auch die Reaktionsflexibilität, d.h. die schnelle Abarbeitung eingehender Aufträge, verstanden. Die Reaktionsflexibilität stellt insbesondere im Bereich der Kommissionierung nach dem Ware-zum-Mann Prinzip eine besondere Herausforderung dar. Nachfolgend wird analysiert, wie die Einflussfaktoren:

- Transparenz

- Reaktionsflexibilität

- Einsatzflexibilität

die Auswahl der Lagertechnik für einen bestimmten Einsatzfall beeinflussen und durch innovative Ansätze beherrschbar werden.

\section{Transparenz}

Der Faktor Transparenz stellt eine wesentliche Voraussetzung für effizienten Lagerbetrieb dar. Ein Schlüsselelement eines Warehouse Managementsystems (WMS) ist die Vermittlung von Vertrauen und Sicherheit in das Führungs- und Kontrollsystem des Lagers. Beispielsweise ist eine wesentliche Ursache für die Anhäufung überhöhter Bestände schlichtweg Unsicherheit auf Seiten der Disponenten oder anderer Lagerverantwortlicher [Schmidt04, Seite 7]. Solche Unsicherheit resultiert u.a. aus einer unvollständigen Datenbasis oder durch zeitraubende Recherchen nach Bestandsmengen, Lagerorten oder Auftragsstati. Ein transparentes System beginnt bei der Datensicherheit und schafft dadurch Vertrauen in die Gültigkeit der Datenbasis. Transparente Abläufe bieten im Lager die wesentliche Grundlage für eine kontinuierliche Systemoptimierung.

Das WMS bedarf zur Erreichung dieser Zielsetzung der eindeutigen Zuordnung von Lagerplatz (Lagerort) und Lagereinheit. Sie dienen der Verwaltung der an einem beliebigen Ort des Lagers aktuellen Menge eines Artikels sowie dessen weitergehende Informationen, wie z.B. Mindesthaltbarkeitsdatum, Chargennummer oder Sperrkennzeichen.

Existierende technische Lagersysteme bieten dazu jedoch höchst unterschiedliche Voraussetzungen. Regalläger besitzen im Allgemeinen eine feste und eindeutige Zuordnung von Regalfach zu Lagerort und somit auch den Bezug von Lagerort zur Lagereinheit. Speziell im Fall der Bodenblocklagerung stellt sich die Situation jedoch anders dar. Eine eindeutige Zuordnung ist zwar theoretisch möglich und wird im Bereich der voll automatisierten Lagersteuerung, bspw. in automatischen Coil- oder Papierlägern, erfolgreich genutzt. Bei staplerbedienten Lägern steht bereits der betriebliche Alltag einer exakten Lagerortverwaltung entgegen. Vor dem Hintergrund, dass Bodenblockläger neben geringer Investitionskosten und hoher Flächennutzung oftmals 
aufgrund ihrer skalierbaren und hochleistungsfähigen Umschlagleistung realisiert werden, ist die Möglichkeit zur präzisen Verfolgung einer einzelnen Lagereinheit sehr begrenzt.

Neuere Lösungsansätze versuchen aufbauend auf der Technologie Fahrerloser Transportsysteme die Staplerposition kontinuierlich zu erfassen und über geeignete Sensorik die Höheninformation im Moment der Einlagerung zur erfassen und in einem Lagerverwaltungssystem zu hinterlegen. Die Steuerung erfolgt in diesem Fall über ein automatisches Staplerleitsystem [NN01]. Andere Lösungen greifen auf andere Erfassungssysteme wie das Global Positioning System (GPS) zurück.

Eine systemtechnische Alternative existiert bislang nur im Einsatz von Ein-/Durchfahrregalen oder Durchlaufregalen. Diese Lösungen besitzen jedoch verschiedene Nachteile hinsichtlich der Raumnutzung (u.a. technisch notwendige Abstände zwischen den Lagerebenen und den Lagersäulen) und/oder der Bedienbarkeit (Staplerfahrten im Lagerkanal eines Durchfahrregales). Ein gänzlich neuer Ansatz in diesem Bereich versucht nun die Merkmale von kompakter Blocklagerung im Stapel mit dynamischen Lagersystemen zu koppeln und dadurch einen Weg zur Automatisierung in diesem, bislang personalintensiven, Bereich zu erschließen. Die vom Fraunhofer Institut für Materialfluss und Logistik (Fraunhofer IML) in Dortmund entwickelte Lösung „Block-It“ verbindet die Hauptmerkmale der Bodenblocklagerung mit denen eines Durchlaufregallagers [Schmidt04]. Wie bei Fließlagersystemen üblich, vereinigt das System die Funktionen „Lagern“ und „Fördern“. Die Lagerung erfolgt zwangsweise nach dem Prinzip „FIFO - First-in First-out“; Einlagerungs- und Auslagerungsseite sind räumlich voneinander getrennt. Im Gegensatz zu den gängigen Durchlaufregallagersystemen werden die Ladeeinheiten (s. Abb. 1, Pos.1) nicht in Kanälen, sondern als Stapel transportiert. Die Ein- und Auslagerung erfolgt entweder durch Frontstapler (halbautomatisches System) oder alternativ durch eine automatische Bedientechnik (vollautomatisches System).

Der Transport der Stapel von der Einlagerungs- zur Auslagerungsseite geschieht mit Hilfe mehrerer paralleler Rollenbahnen (s. Abb. 1, Pos.2). Die Rollenbahnen sind rein passiv ausgeführt, besitzen also keinen eigenen Antrieb. Die Förderbewegung wird durch einen angetriebenen Schlitten (s. Abb. 1, Pos.3) erreicht, der mit einem Mitnehmer ausgerüstet ist. Der Mitnehmer befindet sich dabei immer hinter dem zuletzt in die Bahn eingebrachten Palettenstapel. Bei der Vorwärtsbewegung verdichtet der Schlitten alle auf der Bahn befindlichen Ladeeinheiten zu einem Block. Nach dem Entnehmen bzw. Auslagern des Palettenstapels auf der Auslagerungsseite taktet der Schlitten um eine Palettenteilung vor und füllt damit den Auslagerungsplatz.

Zur Einlagerung in die Bahn bewegt sich der Schlitten rückwärts in Richtung der Einlagerungsseite und unterfährt dabei die neu eingelagerten Ladeeinheiten. Der Mitnehmer rastet dabei hinter dem neu eingelagerten Palettenstapel ein. Auf Anforderung des Bedieners können nun mehrere Stapel eingelagert und anschließend verdichtet werden.

Die Steuerung der Schlitten erfolgt wahlweise entweder dezentral mit einer Speicherprogrammierbaren Steuerung (SPS) für mehrere Bahnen, oder zentral mit einer SPS für das Gesamtsystem und einer Anbindung via Feldbus. Bedingt durch die ohnehin vorhandene Sensorik kann die Belegung der Kanäle auf einfache Art erfasst und an einen Lagerverwaltungsrechner übermittelt werden. Darüber hinaus kann eine platzgenaue Buchung der Ladeeinheiten per Radiofrequenz-Identifikation (RFID) oder Barcode realisiert werden. 


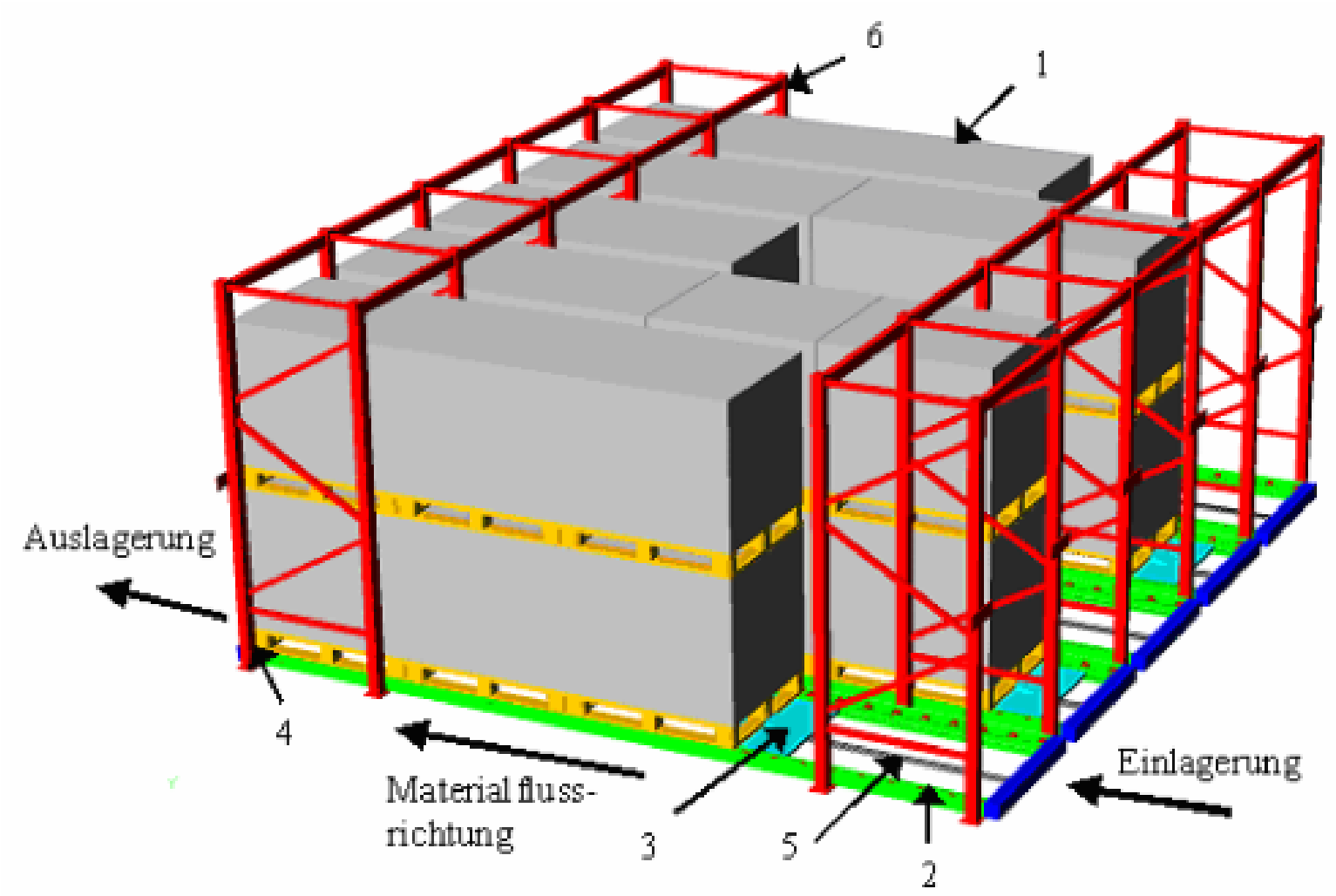

Abbildung 1: Aufbau des automatischen Blocklagersystems

Voraussetzung für den Einsatz des Systems ist eine gute Stapelbarkeit der Ladeeinheiten sowie der Einsatz einheitlicher und rollenbahntauglicher Ladehilfsmittel. Diese Voraussetzungen sind jedoch im Bereich der Bodenblockläger typischerweise gegeben. Aufgrund der Vermeidung von Fahrten im Gang wird eine Steigerung der Umschlagleistung bzw. eine Senkung der Betriebskosten in manuell betriebenen Bodenblocklägern ermöglicht. Bedingt durch die flache Bauweise und die direkte Stapelung der Lagereinheiten ergeben sich im Vergleich zu alternativen Lösungen eine kompaktere Lagerung und ggf. eine zusätzliche Lagerebene. Zudem wird prinzipbedingt ein Verklemmen der Ladeeinheiten - eine im Zusammenhang mit SchwerkraftDurchlaufregalen bekannte Problematik - vermieden. Entscheidend hinsichtlich einer transparenten Lagerverwaltung ist aber die einfache Möglichkeit einer präzisen Verfolgung eingelagerter Einheiten.

\section{Reaktionsflexibilität}

Bei der technischen Entwicklung von Lagersystemen dominierte in den vergangenen Jahren speziell im Bereich leichter Stückgüter der Faktor Ein-/Auslagerleistung, gemessen in Behälter/h bzw. Doppelspiele/h. Neben der Optimierung der Dynamik der Regalbediengeräte, beispielsweise durch Gewichtsoptimierung bewegter Komponenten oder innovativer Antriebskonzepte bis hin zu formschlüssigen Riemenantrieben, wurde die Leistungserhöhung im wesentlichen durch die Erhöhung der Anzahl der Lastaufnahmemittel auf dem Regalbediengerät und somit durch Verringerung der mittleren Anschlussfahrtlänge erreicht. Im Extremfall wird ein Lastaufnahmemittel pro Lagerebene realisiert (System „Sistore“) wodurch Leistungen bis zu 900 Doppelspielen/h ermöglicht werden [Grafe93]. Auch andere Lösungen, z.B. im Bereich der Umlauflagersysteme, vollzogen ähnliche Entwicklungen. Durch Parallelisierung einzelner Bewegungsabläufe können Behälter-Umlaufregalsysteme, mit unabhängigen Lagerebenen ausgestattet, vergleichbare Umschlagleistungen erzielen.

Im Gegensatz zur Durchsatzerhöhung steigt aber auch die auf den einzelnen Behälter bezogene Reaktionszeit des Systems an. Allen durchsatzstarken Systemen gemein ist die Eigenschaft der pulkweisen oder stoßweisen Übergabe der entnommenen Einheiten. Im Fall von Systemen, die zur Lastübergabe einen Stufenlift einsetzen (z.B. „Sistore“) ist darüber hinaus die Auslagerreihenfolge weitgehend festgelegt und kann nur über aufwändige Algorithmen, die bereits bei der Einlagerung greifen, ansatzweise optimiert werden.

Sofern für den Betriebsablauf diese Arbeitsweise, d.h. diskontinuierliche, pulkweise Übergabe mit nicht frei wählbarer Reihenfolge, akzeptabel ist, stellen diese durchsatzstarken Lagersysteme nach wie vor eine interessante Lösung dar. 
Ein solcher Anwendungsfall ist beispielsweise im Bereich der Versandkonsolidierung, d.h. der Zusammenführung von Teilmengen eines Auftrages, die in unterschiedlichen Zonen produziert wurden, gegeben. Ist die Auslagerreihenfolge dagegen nicht beliebig, werden zusätzliche fördertechnische Elemente zur Sortierung erforderlich, die investitionsseitig und hinsichtlich des Flächenbedarfes eine Restriktion darstellen.

Weitergehende Anforderungen an das zeitliche Reaktionsvermögen der Anlage können nur durch Pufferstrecken mit einem daraus resultierenden Flächen- und Investitionsbedarf kompensiert werden. Eine entsprechende Batchberechnung zur Auslager- und Pickreihenfolge ist ebenfalls unumgänglich. Eine solche Batchplanung erfordert aber nicht nur entsprechende Ressourcen (auf entsprechendem Leitsystem implementierte und auf die Aufgabe angepasste Algorithmen) sondern auch Vorlaufzeit zur Schaffung eines für die Optimierung geeigneten Auftragspools.

Bei Betrachtung der Nutzung von Behälterlagersystemen zur Versorgung von Kommissionierstationen im Bereich Ware-zum-Mann, sind an ein Idealsystem daher folgende Anforderungen zu stellen:

- $\quad$ ein leistungsstarkes System mit Auslagerleistungen, die pro Gasse etwa dem Leistungsspektrum einer Kommissionierstation entsprechen (ca. 500-600 Picks/h)

- die schnelle Andienung eines einzelnen Behälters (hohes Reaktionsvermögen)

- $\quad$ eine (nahezu) beliebige Auslagerreihenfolge

- die Fähigkeit, mit dem Leistungsbedarf des Systems zu wachsen (Skalierbarkeit des Systems)

Als Lösungsansatz bieten sich Prinzipien an, die mit einer Vielzahl unabhängiger Einheiten operieren. Eine solche Systemlösung ist das am Fraunhofer-IML entwickelte System „MultiShuttle“. Das Kernelement ist dabei das MultiShuttle-Fahrzeug, ein regalseitig mit Energie versorgtes und mit aktivem Lastaufnahmemittel ausgestattetes Schienenfahrzeug (s. Abb. 2). Im Gegensatz zu bekannten Satellitentechniken wird dabei der fahrzeugseitige Aufwand minimiert, u.a. durch Verlagerung der fahrzeugseitigen Intelligenz auf einen zentralen Rechner und Umgehung einer bordeigenen Energieversorgung.

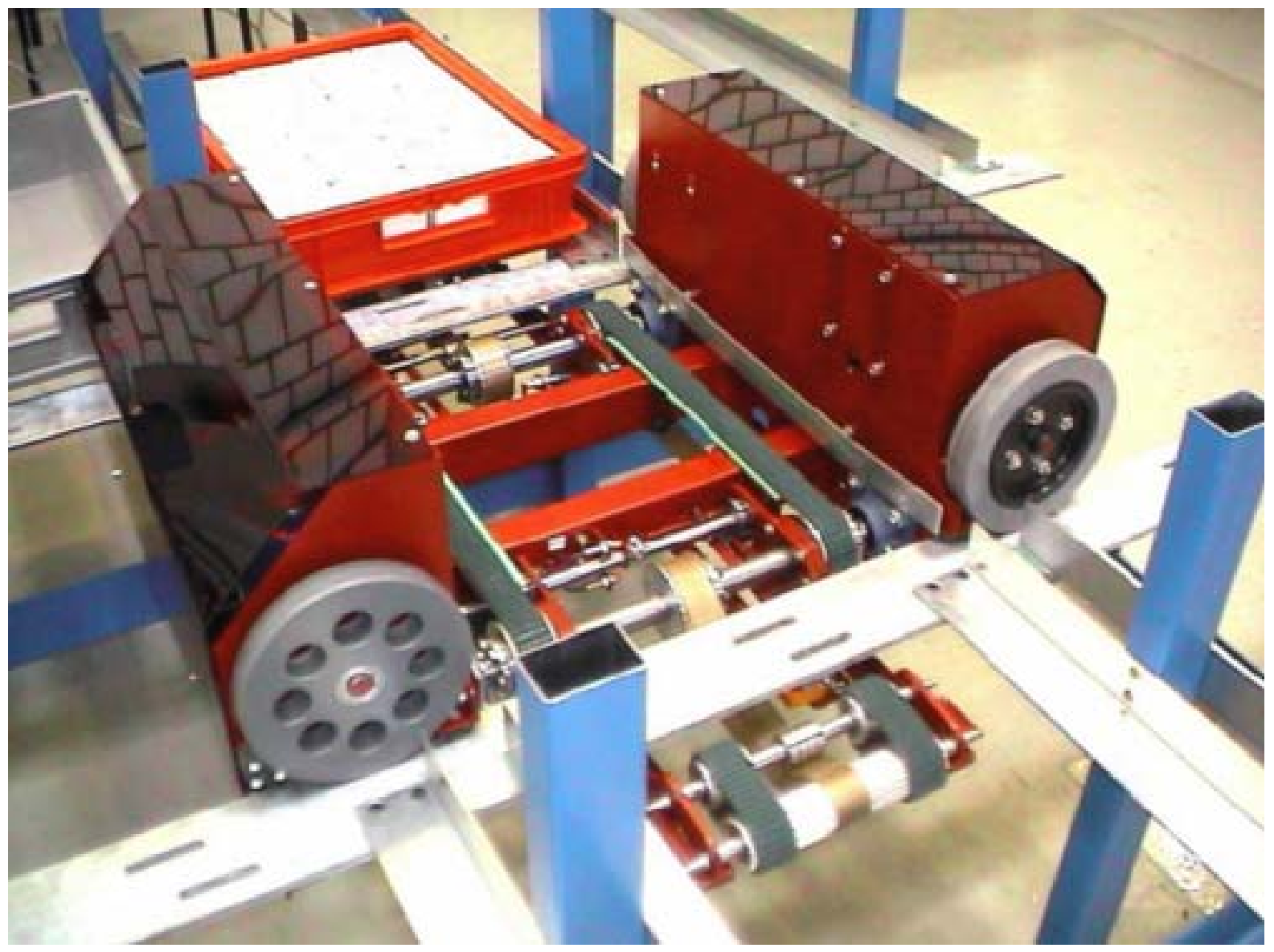

Abbildung 2: - und Lagersystem MultiShuttle

Das Lastaufnahmemittel (LAM) arbeitet mit Reibriemen und kann unterschiedlichste Behälter aufnehmen. Es ragt kontinuierlich seitlich in den Raum unterhalb des aufzunehmenden Behälters, wodurch die bei Regalbediengeräten (RBGs) erforderliche Vorschubbewegung entfallen kann (s. Abb. 3). 
Damit wird nicht nur Platzbedarf, Gewicht und Bauaufwand, sondern auch die Zeit zum Betätigen dieser Bewegungsachse eingespart. Das LAM arbeitet somit prinzipbedingt mit der kürzestmöglichen Spielzeit.

Da die Greifelemente separat bewegt werden können, ist es möglich, an Übergabepunkten schnelle Doppelübergaben durchzuführen. Schiene und LAM sind so flach gebaut, dass sich ein übliches Fachhöhenraster ergibt.

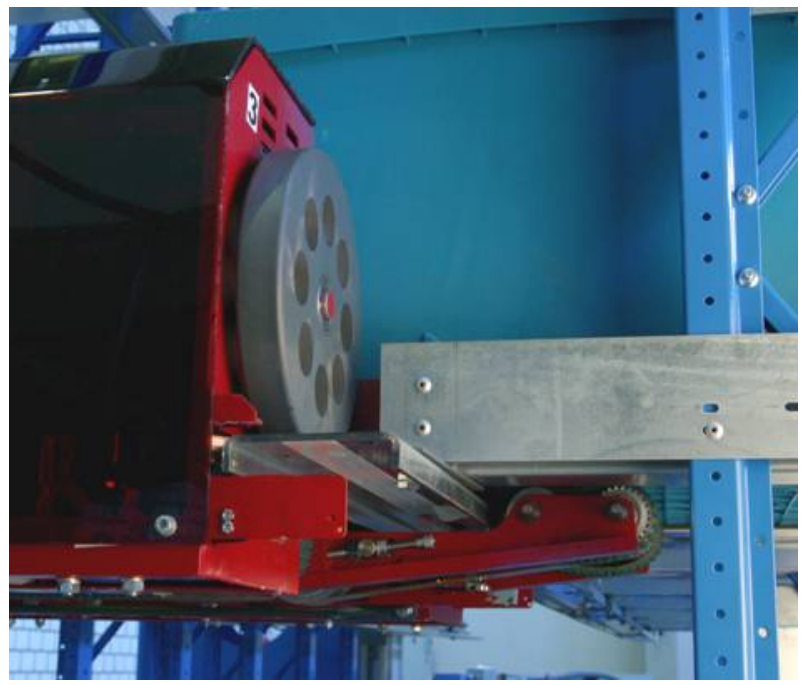

Abbildung 3: Lastaufnahmemittel am System MultiShuttle

Die Fahrschiene ist ein wesentliches Systemelement, in das trotz einfachem Aufbau eine Reihe von Funktionen integriert wurde:

- Tragen und Führen des MultiShuttle

- Energieübertragung

- Positionserfassung und Feinpositionierung

- Schienenende-Absicherung (Herausfall-Sicherung)

Die Schiene ist isoliert an den Winkelauflagen des Regals aufgehängt und mit $24 \mathrm{~V}$ Gleichstrom beaufschlagt. Das MultiShuttle entnimmt seine Energie über die Seitenführungsrollen. Eine netzunabhängige Leistungsversorgung, im innerbetrieblichen Lagerbereich bedeutet dies im Allgemeinen schwere und nachladungskritische Akkumulatoren, sind nicht erforderlich. Die Herausfall-Sicherung arbeitet ohne feste Anschläge oder Stoßdämpfer und verhindert, dass bei einer Fehlfunktion ein MultiShuttle aus dem Regal herausfallen kann.

Im Vergleich zu herkömmlichen Behälterlagersystemen ergeben sich ferner systembedingt folgende weitere Vorteile:

- längere Transportstrecken lassen sich kostengünstig überbrücken, weil die Schiene einfach aufgebaut ist und auch dann, wenn sie flurfrei überkopf montiert wird, keine Wartungsbühnen oder Schutzverkleidungen benötigt

- Stahlblech-Behälter können auch ohne Lärmbelästigung gefördert werden, das ist u.a. im Hinblick auf den Brandschutz vorteilhaft

- $\quad$ im Lagerbereich passt sich das System an die geänderten Anforderungen flexibel an. Bei Steigerungen der Umschlagleistung oder der Stellplatzkapazität ist das System einfach nachrüstbar, so dass das Lager nicht zu Beginn überdimensioniert werden muss. Örtlich oder zeitlich schwankender Leistungsbedarf (z.B. Konzentration der Lageroperationen in einer einzelnen Lagergasse) kann durch entsprechendes Beauftragen der Fahrzeuge ausgeglichen werden

- $\quad$ auch ungünstig geschnittene und nicht quaderförmige Baukörper sind nutzbar

- prinzipbedingt besteht die Möglichkeit, Querstreben zur Versteifung des Regals oder begehbare Böden einzuziehen

\section{Einsatzflexibilität}

Naturgemäß stehen Investitionen in effiziente Lagersysteme und potenzielle Einsparungen im umgekehrten Proporz zueinander. Die Wirtschaftlichkeit einer Investition in ein anforderungsgerechtes Lagersystem erschießt sich somit im Wesentlichen über die Nutzungsdauer. Zunehmend fällt es jedoch schwerer, verlässliche Aussagen über die zukünftige Entwicklung zu treffen. 
Insbesondere der Trend zur Vergabe lagerlogistischer Leistungen an einen externen Dienstleister mit üblichen Vertragslaufzeiten im Bereich weniger Jahre stellt die Planer und Entwickler vor erhebliche Probleme. Immer stärker steht die Forderung im Vordergrund, dass moderne Lagersysteme nach etwaiger Beendigung des Nutzungsverhältnisses für neue Anwendungen nutzbar sind. Je spezieller eine Lösung auf einen Anwendungsfall zurechtgeschnitten ist, desto schwieriger fällt in solchen Fällen diese „Metamorphose“. Üblicherweise werden in Fällen mit unbekannter und begrenzter Nutzungsdauer fixkostenarme Lösungen präferiert, die durch einen hohen Anteil manueller Tätigkeiten geprägt sind.

Im Bereich der Entwicklung lagertechnischner Systeme steht daher zukünftig die Forderung nach möglichst großer Anwendbarkeit und Anpassbarkeit an verschiedenartige Einsatzfälle im Vordergrund. Dazu zählt insbesondere die Adaptierbarkeit auf wechselnde Lagergüter (z.B. Abmessungen, Gewichte, Ladehilfsmittel) und Bewegungsdaten (artikelspezifische Durchsätze, Zugriffe etc.). Ein Lösungspfad ist sicherlich darin zu sehen, Aufwände für nachträgliche Änderungen möglichst umfassend zu erfassen und mit Hinblick auf Änderungskosten und -dauer zu optimieren. Prinzipiell gilt es ferner, bereits im Vorfeld durch geeignete Gestaltung, ein möglichst breites Einsatzfeld zu erschließen. Ein besonderes Beispiel im Bereich der Parksysteme stellen so genannte multifunktionale Parksysteme dar.

In dem Bereich der Anlagen des ruhenden Verkehrs stellen automatische Parksysteme die höchsten Anforderungen an die installierte Technik, da ein eingelagertes Fahrzeug einen Artikel mit der Losgröße 1 darstellt, der permanent mit kurzer Reaktionszeit verfügbar sein muss. Dies wirkt sich sowohl für alle mechanischen und elektrotechnischen Komponenten als auch für den Bereich der Steuerungstechnik und Parkhausverwaltungssysteme aus. Im Hinblick auf die erforderliche Verfügbarkeit ist der Einsatz zuverlässiger und hochwertiger Komponenten notwendig. Die Programmierung der Verwaltungssysteme und Steuerungstechnik erfordert unter anderem die Voraussicht aller möglichen Nutzer- und Bedienerfehler in Verbindung mit einer hohen Ausfallsicherheit des Gesamtsystems. Die Berücksichtigung dieser Forderungen spiegelt sich in den Investitionskosten für diese Systeme wider. Obwohl automatische Parksysteme gegenüber konventionellen Systemen, wie Parkhäusern und Tiefgaragen, einen erheblich besseren Flächen- und Volumennutzungsgrad aufweisen, kann deswegen nicht zwangsläufig eine wirtschaftlich attraktive Lösung generiert werden. Eine Möglichkeit zur Verbesserung der Wirtschaftlichkeit und damit zur Steigerung der Attraktivität automatischer Parksysteme liegt in der Erweiterung des Nutzungsspektrums.

Die Betrachtung der gegenwärtigen Nutzung konventioneller Parkhäuser und Tiefgaragen verdeutlich das Potenzial, welches in diesen Bereichen bei dem Einsatz automatischer Systeme vorhanden ist. Die Verteilung der Stellplatzkapazitäten vermittelt einen ersten Eindruck vorhandener Lagerkapazitäten innerhalb deutscher Städte und Gemeinden.

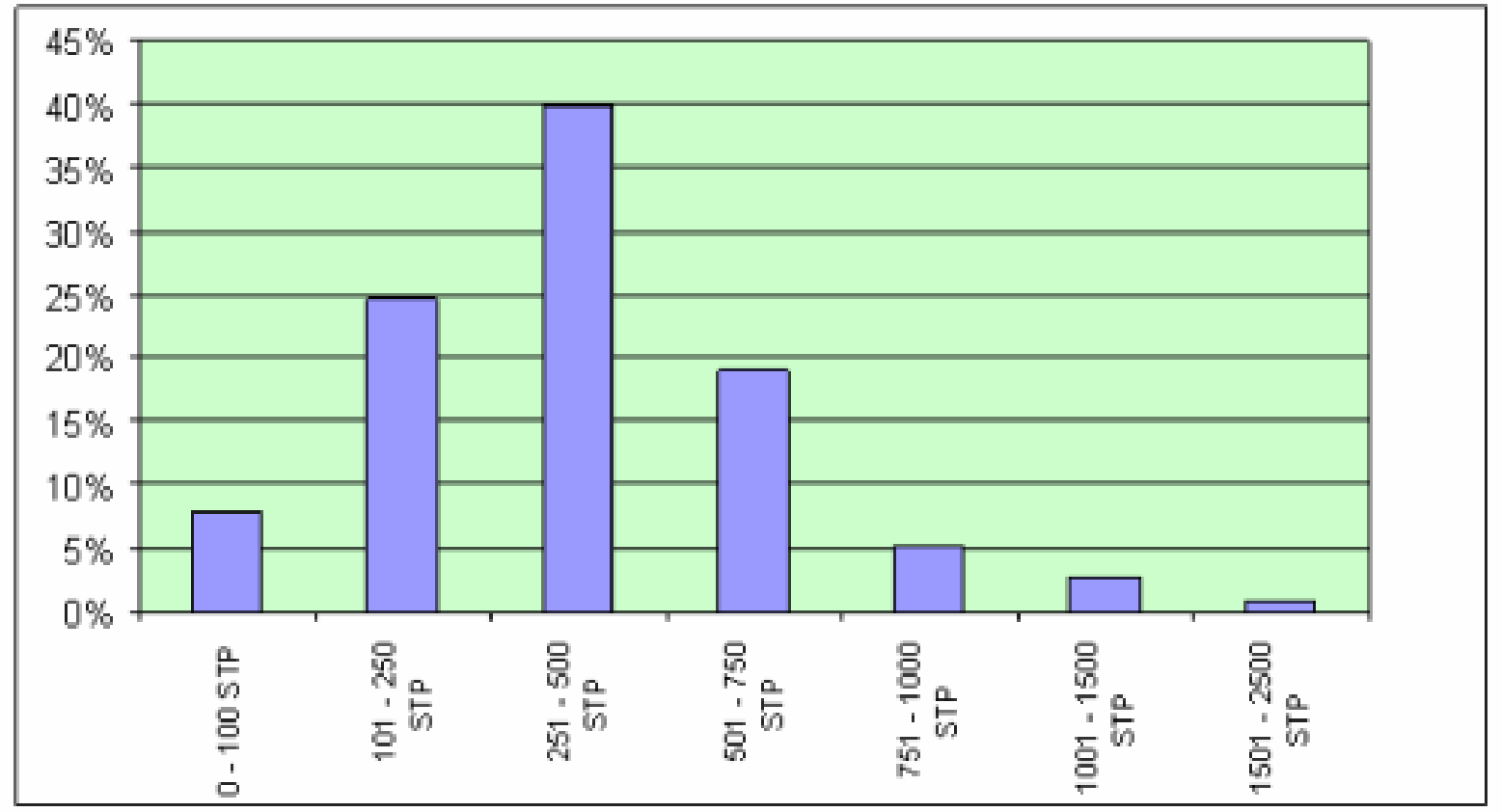

Abbildung 4: Stellplatzverteilung konventioneller Parksysteme in innerstädtischen Bereichen [Kuhn04]

Weitere Gründe zur Errichtung automatischer Parksysteme bzw. multifunktionaler Parksysteme liefert die Darstellung der Öffnungszeiten konventioneller Parkhäuser und Tiefgaragen (Abb. 6). 


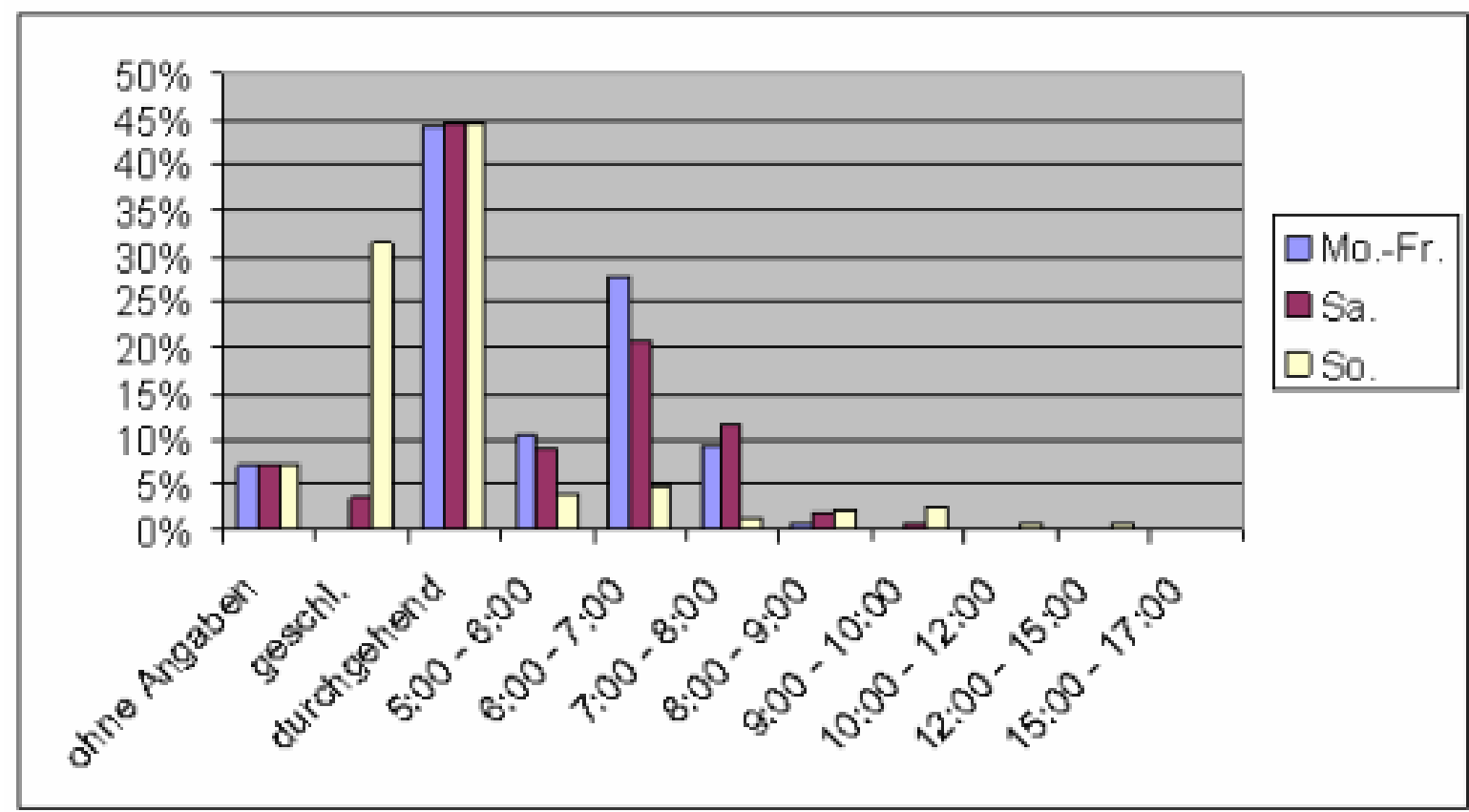

Abbildung 5: Öffnungszeiten konventioneller Parksysteme [Kuhn04]

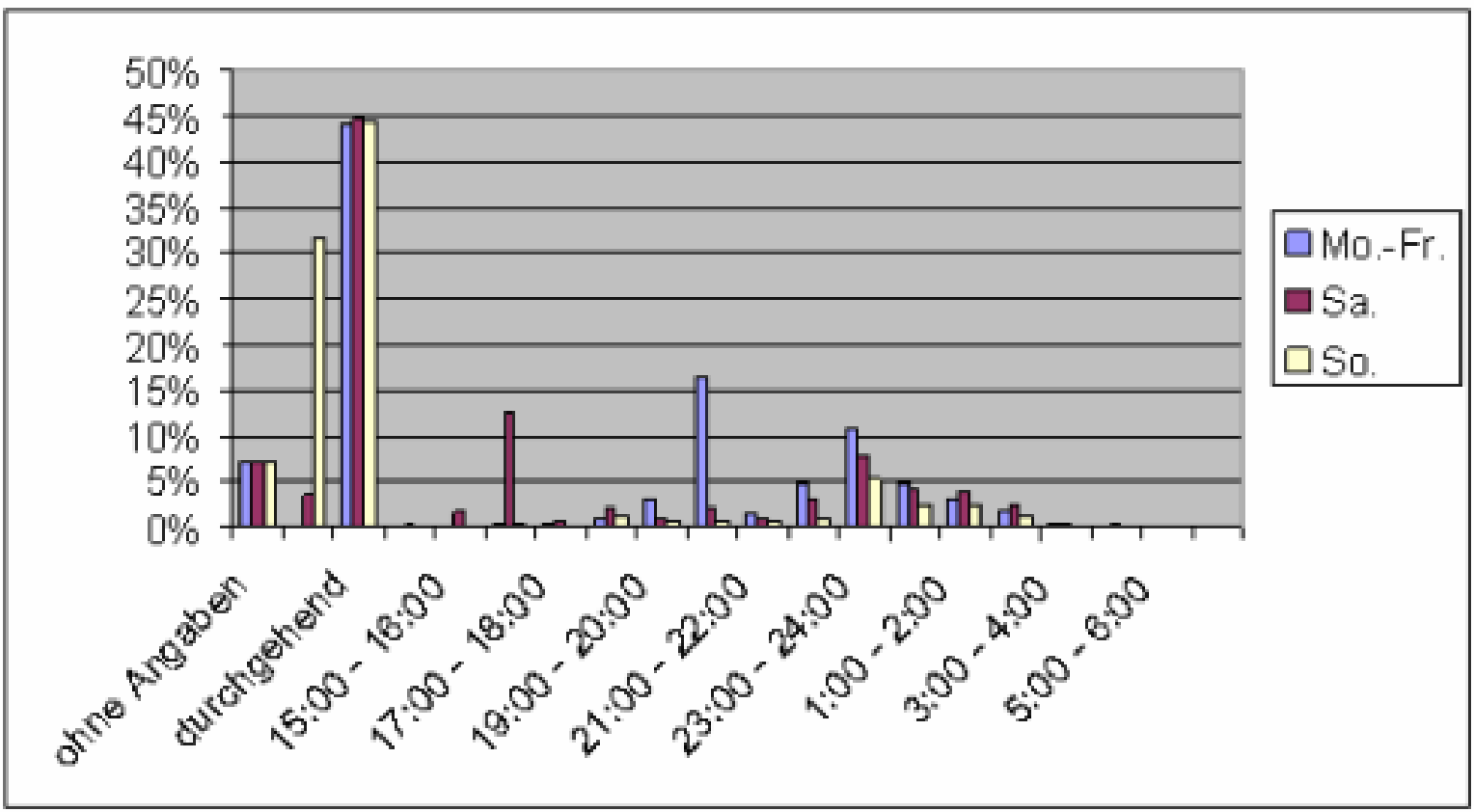

Abbildung 6: Schließungszeiten konventioneller Parksysteme [Kuhn04]

Wie die Analysen zeigen, besteht sowohl hinsichtlich der Nutzungszeiten als auch der Stellplatzkapazitäten ein erhebliches Potenzial zur Nutzung vorhandener Lagerkapazitäten für alternative Lagergüter.

In einer Vielzahl automatischer Parksysteme kommen Roll- oder Gleitpaletten als Ladehilfsmittel zum Einsatz. Die Abmessungen sind anbieter- und systemabhängig und reichen von $2000 \mathrm{~mm}$ bis $2400 \mathrm{~mm}$ in der Breite und 4500 mm als so genannte Radstandspalette bis 5400 mm als Vollpalette in der Länge. Wird diese zur Lagerung bereitgestellte Grundfläche nicht ausschließlich zum Abstellen und Lagern von Pkw genutzt, können automatische Parksysteme in multifunktionale Lagersysteme überführt werden.

Der Ansatz hierzu liegt in der Ausnutzung von Synergieeffekten, welche sich durch die Präsenz von Personenindividual- und Güterverkehr innerhalb bestimmter Gebiete und Knotenpunkte ergeben. Ziel dieses Konzepts ist die zeitgleiche oder zeitversetzte Nutzung vorhandener technischer Einrichtungen und Gewerke zur Lagerung von Pkw und/oder anderen Gütern. Anwender solcher Systeme können in den speziellen Übergabebereichen die bereitgestellte Lagerfläche (Großpalette) oder Teilbereiche dieser (z.B. definierte Zonen, Fächer in speziellen Aufbauten) unter vorgegebenen Randbedingungen zur Lagerung von Pkw oder diversen 
Gütern nutzen. Dieser Ansatz erfordert jedoch eine spezielle Betrachtung aller für eine solche multifunktionale Nutzung relevanten Komponenten.

Bei der Dimensionierung dieser Systeme nehmen, unter Berücksichtigung der zeitlichen Komponente, einzelne Auslegungsparameter unterschiedliche Stellenwerte ein. Müssen bei der zeitgleichen Nutzung bei der Ermittlung der erforderlichen Stellplatzkapazität die Maximalwerte sowohl des Pkw- als auch des Güterbereichs berücksichtigt werden, erfolgt bei der zeitversetzten Nutzung die Dimensionierung naturgemäß nach der Kapazitätsbedarfsprognose des nachfragestärksten Bereichs.

In der vereinfachten Darstellung besteht ein automatisches Parksystem aus

- den Übergabeboxen als Schnittstelle Nutzer-System

- den Förder- und Lagermitteln

- den sicherheitstechnischen Einrichtungen

- dem Steuerungssystem

- dem Parkhausverwaltungssystem.

Es verfügt somit bereits über die wichtigsten Komponenten eines klassischen Lagersystems. Die anforderungsgerechte Adaption der genannten Komponenten auf das erweiterte Einsatzgebiet bzw. die Installation ggf. erforderlicher Zusatzkomponenten stellen die Hauptaufgaben zur Realisierung solcher Systeme dar. Als Einsatzgebiete solcher Systeme können der Business-to-Business und Business-to-Consumer /oder Customer-Bereich aber auch konventionelle Lager- bzw. Pufferfunktionen angeführt werden.

Exemplarisch wird nachfolgend ein Konzept eines multifunktionalen Lagersystems für den innerstädtisch Bereich zur Verdeutlichung des Ansatzes aufgezeigt (s. Abb. 7). Ein multifunktionales Lagersystem stellt hier die Kombination eines Güter-/Warenlagers für den innerstädtischen Einzelhandel und einer Parkierungsanlage für die verschiedenen, in den Innenstädten auftretenden Nachfragegruppen dar. Die zentrale Bereitstellung flexibler Lagerkapazitäten für den Einzelhandel führt zu einer Verringerung der Anlieferfrequenzen, ferner werden hier Synergien durch die räumliche Zentralisierung der Anlieferzielorte genutzt. Das Systemkonzept setzt hier auf ein zentrales, multifunktionales Lagersystem innerhalb eines Einkaufszentrums. Durch die Anbindung der einzelnen Einzelhandelsunternehmen an dieses „Zentrallager“ ist die individuelle Nachschubversorgung sichergestellt. Die Einrichtung zielnaher Kundenparkplätze ist als weiteres Argument anzuführen. Neben dem Einsatz in Einkaufszentren ist die Platzierung multifunktionaler Lagersysteme in Bürogebäuden möglich. Neben der Einrichtung von Mitarbeiterstellplätzen ist hier die Realisierung eines 24Stunden-Anliefer-Konzepts realisierbar. Ferner können in diesem System Lagerplätze als Archiv oder Ausstattungslager genutzt werden. Ähnliche Konzepte sind als Teil eines City-Logistik-Umschlaglagers, als kombiniertes Lager eines Industriebetriebs oder als gemeinsames Lager und Parkhaus in einem Gewerbegebiet oder Gewerbepark denkbar.

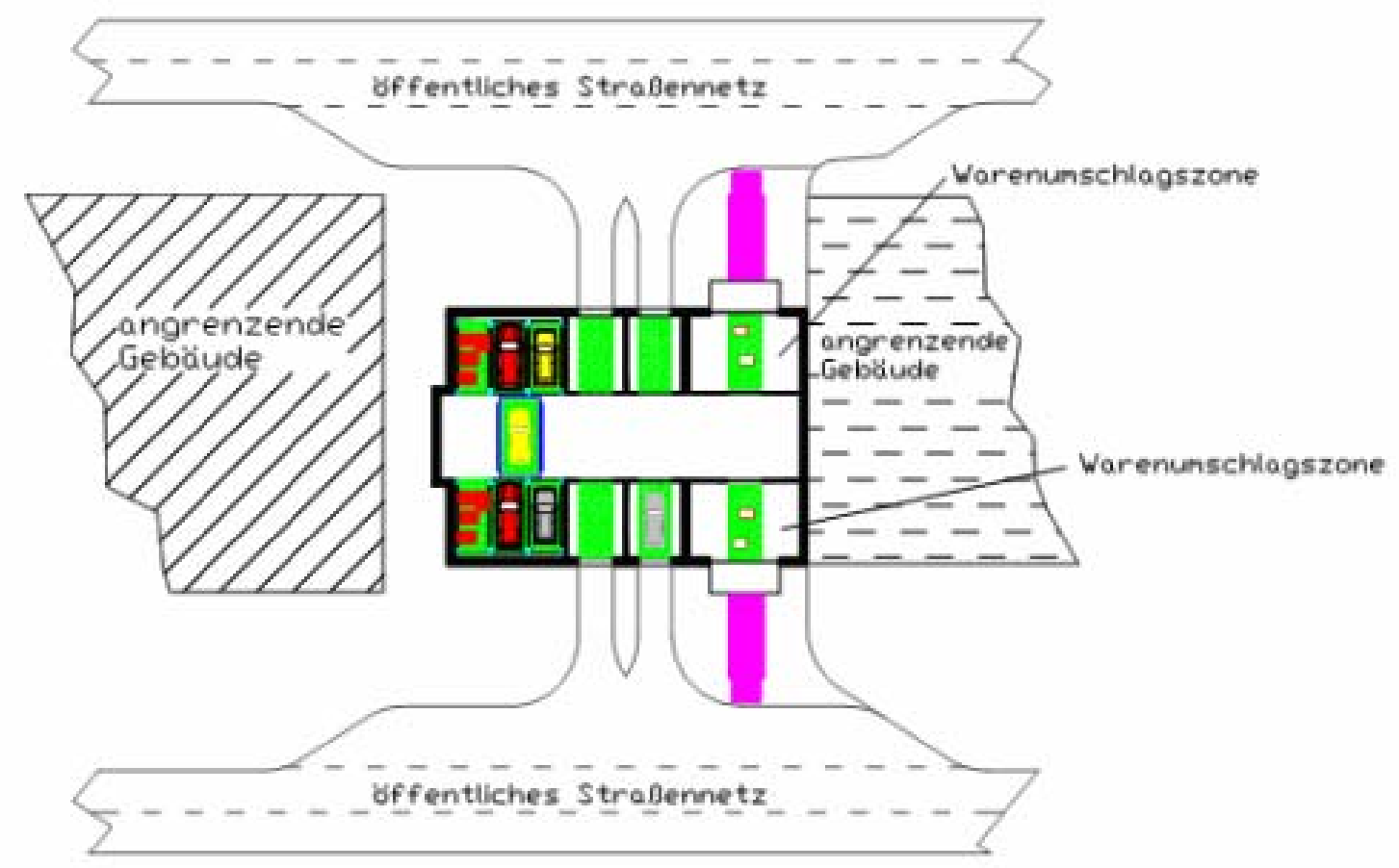

Abbildung 7: Layoutentwurf Innenstadt 
Während heutige automatische Parksysteme die technische Basis für multifunktionale Anwendungen darstellen, sind für eine wirtschaftlich erfolgreiche Nutzung einsatz-, standort- und systemspezifischer Randbedingungen zu berücksichtigen und bei der Gestaltung der Systeme einzubeziehen. Dazu zählen insbesondere:

- die Übergabebox mit angepassten Grundabmessungen und Vermessungseinrichtungen

- $\quad$ eine anforderungsgerechte Gestaltung der Bodenfläche, inkl. ausreichend zulässiger Flächenbelastung

- angepasste sicherheitstechnische Einrichtungen

- die erforderliche Erweiterung der Ein- und Ausgabegeräte (Schnittstellen des Lagerverwaltungssystems)

- $\quad$ eine funktionsgerechte Gestaltung der Zufahrtsbereiche

Werden weitere Forderungen an den Lagerbereich gestellt, wie zum Beispiel gekühlte oder tiefgekühlte Containerfächer, sind in der Übergabebox entsprechende Energieversorgungsanschlüsse vorzusehen. Spezielle Einrichtungen, die während des Übergabevorgangs eine permanente Gewichtskontrolle durchführen, sind für die multifunktionalen Lagersysteme zwingend erforderlich.

Im Bereich der Förder- und Lagertechnik können vorhandene Techniken bestehender automatischer Parksysteme eingesetzt werden. Die gesamte Fördertechnik, deren Lastaufnahmemittel über definierte Schnittstellen zu den Großpaletten verfügen, muss auf die maximalen Abmessungen und das zulässige Maximalgewicht ausgelegt werden. Die Maximalabmessungen sowie das zulässige Maximalgewicht können dabei in bestimmten Anwendungsfällen die Werte bisheriger automatischer Parksysteme überschreiten. Über die Installation einer gesonderten Energieversorgung für die Lagercontainer ist im Einzelfall zu entscheiden. Eine weitergehende Analyse zur Auslegung multifunktionaler Parksysteme liefert [Kuhn04].

\section{Fazit}

Lagersysteme werden zukünftig flexibel im Hinblick auf die Art der Nutzung (Einsatzflexibilität) als auch auf die Abarbeitung der eingehenden Aufträge (Reaktionsflexibilität) auszulegen sein. Hinzu kommen gestiegene Anforderungen die Transparenz der Abläufe als Grundlage für eine umfassende, automatische Lagerverwaltung. Diese Anforderungen stellen nicht nur entsprechende Anforderungen an die Funktionalität eines zu installierenden Lagerleitsystems (WMS) sondern auch an die systemtechnische Gestaltung der Lagertechnik. Im Beitrag wurden dazu verschiedene Lösungsansätze analysiert.

\section{Literatur}

[Grafe93] Grafe, Wolfram: Gestaltung und Betrieb von Umschlaglägern mit hohen Umschlagleistungen, Dissertation. Praxiswissen Verlag, 1994

[Jünemann94]

[Kuhn04]

[NN01]

[Schmidt04]

[TenHompel04] Jünemann, Reinhardt: Neue verkehrslogistische Konzepte für eine ökologisch verantwortbare Transportwirtschaft, Dhf, (1994) 6, S. 176 - 181

Kuhn, Markus: Beitrag zur Gestaltung multifunktionaler Lagersysteme, Dissertation, Praxiswissen Verlag, 2004

N.N.: Gerolsteiner positioniert sich neu-Automatische Chargenverfolgung, Logistik Heute, 23 (2001) 6, S. 20-22

Schmidt, Thorsten; Könemann, Matthias; ten Hompel, Michael: Block-it für palettierte Stückgüter, Hebezeuge und Fördermittel, (2004) 10, S. 540-542 ten Hompel, Michael; Schmidt, Thorsten: Warehouse Management - Organisation und Betrieb von Kommissionier- und Lagersystemen, 2. Auflage, Springer-Verlag, 2004 